\title{
Persistência de plantas medicinais em sistemas agroflorestais no município de São Bento do Sul, SC, Brasil
}

\author{
HANISCH, A.L. ${ }^{1}$; PESSETTE, S.C.S. ${ }^{2}$; SCHMITT L.L. ${ }^{3}$; BAVARESCO, A. ${ }^{4}$, FONSECA, J.A. ${ }^{5}$ \\ 1, ${ }^{5}$ Epagri - Estação Experimental de Canoinhas, Cx. Postal 216, CEP 89460-000 Canoinhas/SC. E-mail:

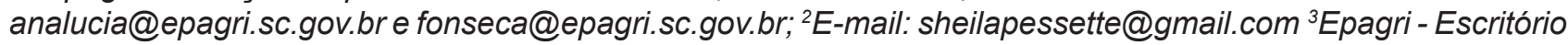 \\ Municipal de São Bento do Sul - e-mail: luluiza@epagri.sc.gov.br; ${ }^{4}$ Ministério da Agricultura
}

RESUMO: No final da década de 90, a comunidade de Rio Natal, no município de São Bento do Sul, SC, passou a fazer parte de uma Área de Preservação Ambiental e desde então, muitas áreas de cultivo, paulatinamente, estão retomando o processo de formação florestal. Neste cenário, foi sugerido pela comunidade, a possibilidade de cultivo de plantas medicinais adaptadas às condições de sombreamento. Em atendimento a essa demanda foi realizada uma pesquisa participativa na comunidade, de 2005 a 2008, onde foram avaliadas 14 espécies de plantas medicinais com potencial de mercado, cultivadas em áreas sombreadas, em três propriedades familiares. Foram avaliados: taxa de sobrevivência das espécies, incidência de doenças e pragas, teor de massa seca e altura das plantas. Apresentaram persistência ao cultivo em áreas sombreadas as espécies: Curcuma longa L. (açafrão-da-índia); Arctium lappa L. (bardana); Pfaffia glomerata (Spreng) Pedersen (fáfia ou ginseng-brasileiro); Mikania glomerata Sprengel (guaco); Alternanthera brasiliana L. Kuntze (penicilina); Pogostemon cablin (Blanco) Benth (patchuli) e Maytenus ilicifolia Mart. Ex Reissek (espinheira-santa).

Palavras-chave: agroecologia, Floresta Ombrófila Densa, pesquisa participativa

\begin{abstract}
Persistence of medicinal plants in agroforestry systems in the city of São Bento do Sul, Santa Catarina, Brazil. In the end of the 1990s, the Rio Natal community, located in the city of São Bento do Sul, in the Brazilian State of Santa Catarina, started to be part of an Environmental Protection Area. Since then, many cultivation areas have been increasingly having their forest restored. In this scenario, the community proposed to cultivate medicinal plants that were adapted to shaded conditions. To attend this demand, we carried out a participatory research in the community between 2005 and 2008. Fourteen species of medicinal plants having market potential were evaluated, and were cultivated in shaded areas, in three family properties. The survival of the species, the incidence of illnesses and pests, the dry matter value and the height of the plants were evaluated. The species that presented persistence to be cultivated in shaded areas were: Curcuma longa L.; Arctium lappa L.; Pfaffia glomerata (Spreng) Pedersen.; Mikania glomerata Sprengel.; Alternanthera sp.; Pogostemon cablin (Blanco) Benth. e Maytenus ilicifolia Mart. Ex Reissek.
\end{abstract}

Keywords: agroecology, Dense Ombrophylous Forest, participatory research

\section{INTRODUÇÃO}

Atualmente, em face ao processo de mudanças globais resultante da crescente degradação ambiental, é de grande relevância a criação e manutenção de áreas voltadas à conservação e preservação dos recursos naturais. No Brasil, as Áreas de Proteção Ambiental (APAs) compõe o grupo das Unidades de Uso Sustentável conforme o Sistema Nacional de Unidades de Conservação - lei no 9.985/2000 (BRASIL, 2000). De acordo com a lei, nessas áreas, a atividade humana pode e deve existir, desde que orientada e regulada de forma a proteger a diversidade biológica e garantir o uso sustentável dos recursos naturais, além de disciplinar o processo de ocupação do meio. A APA de Rio Vermelho/Humboldt no município de São Bento do Sul em Santa Catarina foi fundada em 1998, com uma área de 23.000 ha, mais da metade da área total do município. A comunidade de Rio Natal faz parte desta APA e, desde então, os agricultores vem buscando mudar as atividades 
produtivas, uma vez que os antigos métodos de cultivo, que consistiam, inclusive, em desmatamento e queimada, foram proibidos, reduzindo as áreas de cultivo, que pouco a pouco estão retomando o processo de formação florestal. Neste cenário, os trabalhos de extensão rural em parceria com a comunidade, levantaram a possibilidade do cultivo de plantas medicinais com potencial de mercado nas áreas de recuperação ambiental das propriedades, na busca de associar a conservação ambiental a uma estratégia de geração de renda para as famílias. O manejo sustentável dos recursos naturais é considerado como uma estratégia eficiente para a manutenção da biodiversidade das florestas, na medida em que pode possibilitar retorno econômico a comunidades rurais, aliado à prática da conservação (Steenbock, 2003).

Uma das premissas desta pesquisa era de que fosse realizada através de um processo participativo. A pesquisa participativa é uma estratégia que tende a melhorar o planejamento, a condução, a avaliação, a interpretação dos resultados e a difusão do conhecimento gerado, inclusive a partir de critérios adotados com rigor metodológico. Seu uso implica em maior comprometimento dos vários segmentos envolvidos, além de promover a adoção ou não, quase imediata, dos resultados gerados (Hanisch \& Meister, 2009).

Um dos grandes desafios do manejo sustentável em áreas de floresta é o comportamento produtivo das espécies comerciais em relação à redução da intensidade luminosa. A adaptação da estrutura interna das folhas, no período de crescimento, aos diferentes níveis de luz do ambiente é considerada uma plasticidade adaptativa comum a espécies que apresentam amplo potencial de aclimatação (Whatley \& Whatley, 1982). Essa plasticidade estrutural está associada a uma função compensatória de folhas adaptadas à sombra, à diminuição proporcional da fotossíntese e à diminuição da intensidade luminosa, visto que tais folhas aproveitam melhor a luminosidade, em comparação com aquelas não adaptadas a esse fator (Larcher, 2000).

Devido à pouca informação disponível sobre a influência da redução da luminosidade na produção de espécies medicinais em sistemas agroflorestais, é importante a avaliação da adaptação através da taxa de sobrevivência de espécies comerciais nestes ambientes. $O$ objetivo deste trabalho foi $\mathrm{O}$ de avaliar a taxa de sobrevivência e características produtivas de espécies de plantas medicinais em áreas sombreadas, que podem ocorrer em áreas de regeneração e/ou remanescentes florestais.

\section{MATERIAL E MÉTODO}

$O$ experimento foi desenvolvido em três propriedades rurais da Comunidade de Rio Natal no município de São Bento do Sul (26¹4' S e 49²2' W). A altitude média das três áreas é de $1.000 \mathrm{~m} \mathrm{e}$ a vegetação natural é de Floresta Ombrófila Densa, apresentando como espécies mais importantes árvores da família das Lauráceas, além de muitas Mirtáceas e com o estrato médio constituído, por um número relativamente pequeno de árvores medianas, dentre as quais se destaca o palmiteiro (Euterpe edulis) (Schäffer \& Prochnow, 2002).

O clima da área de estudo é do tipo Cfa (mesotérmico úmido de verão quente) segundo a classificação de Köppen, com temperatura média do mês mais quente superior a $18^{\circ} \mathrm{C}$, apresentando uma precipitação média anual em torno de 1.500 $\mathrm{mm}$. O relevo predominante na área enquadra-se na classe forte ondulado e montanhoso.

Foram selecionadas três propriedades rurais em função da sua semelhança em relação à densidade da vegetação, ao interesse da família em se envolver na pesquisa e em função da sua localização na comunidade. Em cada uma das três propriedades foi marcada uma área amostral de aproximadamente $1.000 \mathrm{~m}^{2}$, definidas de acordo com a localização e disponibilidade de terreno nas propriedades. As áreas foram denominadas em ordem crescente em função da declividade do terreno, de $5 \%, 15 \%$ e $30 \%$, respectivamente para as áreas 1,2 e 3 . A escolha das áreas com declividades diferentes foi uma solicitação da comunidade a fim de analisar se em áreas com maior declive haveria condições de serem executados as colheitas e os tratos agronômicos necessários ao desenvolvimento das plantas. Algumas características químicas e físicas dos solos das áreas experimentais estão descritos na Tabela 1.

A escolha das espécies de plantas medicinais se deu a partir de três critérios: revisão bibliográfica sobre plantas cultivadas em

TABELA 1. Caracterização parcial dos solos das três áreas experimentais em Rio Natal, São Bento do Sul/SC, 2005.

\begin{tabular}{cccccccc}
\hline Localização & $\begin{array}{c}\mathrm{pH} \\
\text { (água) }\end{array}$ & $\begin{array}{c}\text { Argila } \\
\%\end{array}$ & $\begin{array}{c}\mathrm{V} \\
\%\end{array}$ & $\begin{array}{c}\mathrm{M} . \mathrm{O} \\
\%\end{array}$ & $\begin{array}{c}\mathrm{Al} \\
\mathrm{cmol} / \mathrm{dm}^{3}\end{array}$ & $\begin{array}{c}\mathrm{K} \\
\mathrm{mg} / \mathrm{dm}^{3}\end{array}$ & $\begin{array}{c}\mathrm{P} \\
\mathrm{mg} / \mathrm{dm}^{3}\end{array}$ \\
\hline Área 1 & 5,7 & 26 & 54,6 & 7,4 & 0 & 157 & 49 \\
Área 2 & 5,6 & 30 & 77,5 & 3,2 & 0 & 118 & 8,3 \\
Área 3 & 4,9 & 30 & 43,4 & 4,9 & 1,3 & 67 & 6,4 \\
\hline
\end{tabular}

Rev. Bras. PI. Med., Campinas, v.15, n.4, p.774-779, 2013. 
ambientes com menor luminosidade; indicação de pesquisadores e empresas comerciais de plantas medicinais e realização de um diagnóstico com as três famílias envolvidas, para levantar as plantas com as quais já tivessem tido experiência em áreas sombreadas ou próximas a essas. A partir desse processo foram selecionadas 14 espécies: Curcuma longa L. (açafrão-da-índia); Arctium lappa L. (bardana); Lippia alba (Mill.) N.E. Br. (ervacidreira); Pfaffia glomerata (Spreng) Pedersen. (fáfia ou ginseng-brasileiro); Mikania glomerata Sprengel. (guaco); Artemisia absinthium L. (losna); Malva sylvestris L. (malva); Ocimum basilicum L. (manjericão); Achillea millefolium L. (mil-folhas); Alternanthera brasiliana L. Kuntze (penicilina); Mentha pulegium L. (poejo); Pogostemon cablin (Blanco) Benth. (patchuli); Centaurium umbellatum Gilib. (fel-da-terra); Maytenus ilicifolia Mart. Ex Reissek. (espinheira-santa).

O experimento foi instalado entre novembro/2005 e janeiro/2006. Em cada área experimental foram plantadas de 8 a 20 mudas de cada espécie, em função da disponibilidade de mudas. Para facilitar o acesso às plantas, as mesmas foram agrupadas em dez locais diferentes dentro da área experimental, denominados 'estações', sendo plantado, no mínimo, uma muda de cada espécie/ estação. As estações, por sua vez, foram marcadas com estacas numeradas e registradas em um croqui.

As mudas utilizadas foram provenientes da Epagri - Estação Experimental de Itajaí e de produtores comerciais certificados pela IMO Control do Brasil. Todas as mudas estavam acondicionadas em sacos plásticos $(12 \times 8 \mathrm{~cm})$, com substrato comercial para mudas até o momento de serem transplantadas para as áreas experimentais. A espinheira santa foi plantada apenas em novembro de 2006, uma vez que as mudas foram provenientes de sementes selecionadas à época do início do experimento, em 2005.

As covas foram adubadas com $50 \mathrm{~g}$ de fosfato natural, $500 \mathrm{~g}$ de calcário dolomítico e $5 \mathrm{~kg}$ de cama de aviário $(\mathrm{pH}=8,3$; M.S. $=85,49 \% ; \mathrm{N}=30,45$ $\mathrm{g} / \mathrm{kg} ; \mathrm{P}=14,80 \mathrm{~g} / \mathrm{kg} ; \mathrm{K}=23,10 \mathrm{~g} / \mathrm{kg} ; \mathrm{Ca}=39,53$ $\mathrm{g} / \mathrm{kg} ; \mathrm{Mg}=5,58 \mathrm{~g} / \mathrm{kg}$ ), na ocasião do plantio e em cobertura um ano após. As três áreas experimentais foram roçadas sempre que necessário para permitir o acesso às estações, e as plantas foram mantidas livres de invasoras através de arranquio das mesmas, antes das avaliações.

A partir de março de 2006, foram iniciadas as avaliações relativas à sobrevivência das plantas e resistência a insetos e doenças. A taxa de sobrevivência era avaliada a cada 60 dias no $1^{\circ}$ ano e a cada 120 dias no $2^{\circ}$ ano, através da contagem das plantas vivas/estação. As observações sobre incidência de doença e de insetos foram realizadas no primeiro ano, em parceria com as famílias, que avaliavam as áreas todas as semanas durante os seis meses iniciais e anotavam em uma planilha 0 aparecimento de insetos e as condições climáticas que pudessem beneficiar o aparecimento de doenças. As mudas de espinheira-santa foram protegidas do ataque de formigas por embalagens PET transparentes, cortadas ao meio, pois já havia conhecimento entre os agricultores de que as formigas atacavam severamente essa espécie.

Em fevereiro de 2008 foi medida a altura total $(\mathrm{H})$ de todas as plantas medicinais ainda presentes nas áreas experimentais, sendo medidas da superfície do solo até o ápice da planta, com auxílio de uma régua graduada.

TABELA 2. Número de indivíduos iniciais $(\mathrm{N})$ e taxa de sobrevivência ( $\mathrm{S} \%$ ) de plantas medicinais cultivadas em três áreas de Floresta Ombrófila Densa, após um ano da implantação. Rio Natal - São Bento do Sul, SC, 2007.

\begin{tabular}{|c|c|c|c|c|c|c|c|c|}
\hline \multirow[t]{2}{*}{ Espécies } & \multirow[t]{2}{*}{ Nome comum } & \multicolumn{2}{|c|}{ Área 1} & \multicolumn{2}{|c|}{ Área 2} & \multicolumn{2}{|c|}{ Área 3} & \multirow{2}{*}{$\begin{array}{c}\text { Média } \\
\mathrm{S} \%\end{array}$} \\
\hline & & $\mathrm{N}$ & $\mathrm{S} \%$ & $\mathrm{~N}$ & $\mathrm{~S} \%$ & $\mathrm{~N}$ & $\mathrm{~S} \%$ & \\
\hline Curcuma longa L. & açafrão-da-india & 8 & 50 & 5 & 20 & 5 & 20 & 30 \\
\hline Arctium lappa L. & bardana & 10 & 100 & 8 & 100 & 5 & 100 & 100 \\
\hline Lippia alba (Mill.) N.E. & erva-cidreira & 18 & 50 & 10 & 50 & 10 & 50 & 50 \\
\hline Pfaffia glomerata (Spreng) & fáfia/ginseng-brasileiro & 10 & 90 & 13 & 100 & 10 & 100 & 97 \\
\hline Centaurium umbellatum Gilib. & fel da terra & 8 & 75 & 10 & 0 & 8 & 25 & 33 \\
\hline Mikania glomerata Sprengel & guaco & 20 & 100 & 18 & 100 & 20 & 100 & 100 \\
\hline Artemisia absinthium L. & losna & 10 & 0 & 8 & 0 & 10 & 0 & 0 \\
\hline Malva sylvestris L. & malva & 10 & 10 & 10 & 10 & 10 & 10 & 10 \\
\hline Ocimum basilicum L. & manjericão & 10 & 10 & 10 & 10 & 10 & 10 & 10 \\
\hline Achillea millefolium L. & mil-folhas & 10 & 0 & 10 & 0 & 10 & 0 & 0 \\
\hline Alternanthera brasiliana L. Kuntze & penicilina & 10 & 90 & 8 & 100 & 10 & 60 & 83 \\
\hline Mentha pulegium L. & poejo & 15 & 20 & 15 & 20 & 20 & 15 & 18 \\
\hline Pogostemon cablin (Blanco) & patchuli & 10 & 100 & 13 & 100 & 20 & 50 & 83 \\
\hline Maytenus ilicifolia Mart. & espinheira-santa & 20 & 100 & 20 & 100 & 20 & 100 & 100 \\
\hline
\end{tabular}

Rev. Bras. PI. Med., Campinas, v.15, n.4, p.774-779, 2013. 


\section{RESULTADO E DISCUSSÃO}

O resultado da taxa de sobrevivência das plantas medicinais introduzidas nas áreas de floresta, após um ano de avaliação, pode ser visto na Tabela 2. Das 14 espécies inicialmente presentes, apenas seis mantiveram valores acima de $50 \%$ de sobrevivência após um ano.

Das espécies com maior número de indivíduos em todas as áreas, destacaram-se a Mikania glomerata (guaco) e Arctium lappa (bardana) com $100 \%$ de sobrevivência nas 3 áreas avaliadas. Houve diferenças entre o desenvolvimento das plantas dentro e entre as áreas, mas nenhuma das plantas introduzidas morreu no primeiro ano. A espinheira-santa aparece com $100 \%$ de sobrevivência, mas estava presente na área havia apenas três meses quando foi realizada a avaliação de um ano das demais espécies, o que não permite compará-la com as demais.

O guaco é uma planta perene e trepadeira, que cresce espontaneamente em matas primárias e é esciófita (Silva, 2006). Essa espécie foi beneficiada pela sombra e pela facilidade de encontrar árvores e galhos para expandir seu crescimento. Sabe-se que o cultivo de espécies de guaco a pleno sol aumenta a produção de cumarina, principal princípio ativo dessa planta (Rehder et al., 1998). Para esse autor, as plantas expostas diretamente ao sol são induzidas ao florescimento intenso, acarretando grande dispêndio de energia e declínio no vigor da cultura, a qual vai diminuindo a sua produtividade de ramas e folhas a cada safra. Com relação ao seu desenvolvimento na sombra, Castro et al. (2007) verificaram que as plantas de guaco apresentam adaptabilidade maior a locais de sombreamento moderado, em torno de $30 \%$ de sombra, confirmando os resultados observados neste trabalho.

A bardana cresce espontaneamente nos campos, sub-bosques e áreas ruderais. Sua aclimatação é tamanha, que a planta é considerada planta invasora (Lorenzi \& Matos, 2002) Essa espécie sobreviveu com taxa de $100 \%$ no ambiente sombreado, um ano após a implantação do experimento. Apesar de não ter sido avaliado, o nível de sombreamento variava durante o dia, de acordo com a posição do Sol, em todas as áreas, permitindo o acesso à luz solar em determinadas horas do dia para a maioria das plantas, o que deve ter contribuído para a sobrevivência de espécies como a bardana, que não são exclusivamente de sombra.

Das demais espécies que mantiveram altas taxas de sobrevivência, o patchuli e a penicilina destacaram-se pela ramificação, sendo que em média, cada planta dessas espécies apresentava 3 ramos bem desenvolvidos após 12 meses de plantio. A fáfia embora tenha apresentado alto índice de sobrevivência, apresentava plantas com baixo vigor e poucas folhas. Este comportamento também foi observado na erva-cidreira, que manteve vegetando apenas o ramo principal. Este comportamento da fáfia se deve ao fato de ser uma espécie heliófita, apesar de ocorrer com freqüência em mata ciliar (Guerreiro et al., 2009). O que ser observou neste trabalho é que ela vegeta em ambientes de pouca luz, mas não se desenvolve adequadamente.

A baixa sobrevivência das plantas de losna, malva, manjericão, mil-folhas e poejo foi observada já na primeira avaliação, 60 dias após o plantio. Essas plantas foram selecionadas por serem cultivadas pelos agricultores em quintais próximos às casas, mas confirmaram que são plantas que produzem em áreas abertas, sob maior intensidade luminosa. O açafrão, apesar da taxa de sobrevivência de $30 \%$ apresentou plantas vigorosas em todas as áreas.

De um modo geral, a não sobrevivência das espécies não teve relação com ataque de insetos

TABELA 3. Número de indivíduos $(\mathrm{N})$, taxa de sobrevivência $(\mathrm{S})$ e altura média $(\mathrm{H})$ por área, por espécie 26 meses após a implantação do experimento em área de Floresta Ombrófila Mista em Rio Natal/São Bento do Sul/SC (2008)

\begin{tabular}{|c|c|c|c|c|c|c|c|c|c|c|c|}
\hline \multirow[b]{3}{*}{ Espécie } & \multirow[b]{3}{*}{ Nome vulgar } & \multicolumn{3}{|c|}{ Área 1} & \multicolumn{3}{|c|}{ Área 2} & \multicolumn{3}{|c|}{ Área 3} & \multirow{3}{*}{$\begin{array}{c}\mu \\
H \\
(m)\end{array}$} \\
\hline & & $\mathrm{N}$ & $\mathrm{S}$ & $\mathrm{H}$ & $\mathrm{N}$ & $\mathrm{S}$ & $\mathrm{H}$ & $\mathrm{N}$ & $\mathrm{S}$ & $\mathrm{H}$ & \\
\hline & & & $(\%)$ & (m) & & $(\%)$ & $(\mathrm{m})$ & & $(\%)$ & $(\mathrm{m})$ & \\
\hline Curcuma longa L. & açafrão-da-india & 3 & 37 & 0,5 & 0 & 0 & - & 3 & 60 & 0,4 & 0,48 \\
\hline Arctium lappa L. & bardana & 0 & 0 & - & 5 & 62 & 0,6 & 0 & 0 & - & 0,60 \\
\hline Pfaffia glomerata (Spreng) & fáfia & 7 & 70 & 1,6 & 6 & 46 & 1,6 & 1 & 10 & 0,9 & 1,40 \\
\hline \multicolumn{12}{|l|}{ Pedersen. } \\
\hline Mikania glomerata Sprengel. & guaco & 19 & 95 & 2,8 & 16 & 89 & 3,3 & 10 & 50 & 3,4 & 3,20 \\
\hline Alternanthera brasiliana L. Kuntze & penicilina & 10 & 100 & 1,0 & 5 & 62 & 1,4 & 3 & 30 & 1,1 & 1,20 \\
\hline Pogostemon cablin (Blanco) Benth. & patchuli & 10 & 100 & 1,0 & 13 & 100 & 1,2 & 7 & 35 & 1,0 & 1,10 \\
\hline Maytenus ilicifolia Mart. Ex Reissek. & espinheira-santa* & 20 & 100 & 0,4 & 7 & 35 & 0,3 & 15 & 75 & 0,4 & 0,37 \\
\hline
\end{tabular}

*A espinheira santa estava com 16 meses no momento da avaliação. 
e doenças. Na avaliação participativa desses fatores, não foram observadas doenças nas plantas sombreadas e o inseto que mais causou danos às plantas foi a formiga, que atacou, no início do experimento e com maior intensidade, as folhas de guaco, fáfia, erva-cidreira e patchuli.

Dois anos após a implantação do experimento, com exceção do fel-da-terra e da ervacidreira, foram observadas plantas vivas das demais espécies que haviam sobrevivido ao primeiro ano, em todas as áreas, e a medição da altura dessas plantas, indicou desenvolvimento próximo ao padrão de cada espécie, como pode ser visto na Tabela 3 e discutido a seguir.

Foi observado um padrão semelhante de crescimento para o açafrão-da-índia nos dois anos de avaliação nas três áreas: poucas plantas, vigorosas e que apresentaram florescimento. Essa característica está ligada ao fato das folhas secarem durante o inverno, voltando a brotar na primavera (Silva, 2003). Dessa forma, em muitas avaliações foi difícil identificar plantas de açafrão-da-índia, embora seus rizomas ainda pudessem estar vegetando. A altura média encontrada neste trabalho é quase $50 \%$ inferior à altura relatada por Silva (2003) de $1,20 \mathrm{~m}$. Entretanto, como seu interesse comercial está nas raízes - que não foram avaliadas - sua sobrevivência nas áreas de sombra indica um potencial que precisa ser melhor explorado em trabalhos futuros.

A bardana é uma planta bianual, o que justifica o fato de não ter sido observada em duas das três áreas avaliadas, dois anos após o plantio. Sua presença em todas as avaliações anteriores e os resultados obtidos de altura na última avaliação, indicam capacidade de adaptação ao ambiente sombreado. Gassi et al. (2009) observaram altura que variaram de 70 a $130 \mathrm{~cm}$, aproximadamente, de acordo com as doses de adubação. Com relação à fáfia, os resultados em altura estão de acordo com os valores relatados por Silva (2003). Nas observações a campo foi verificado que o crescimento da fáfia em áreas com menor intensidade luminosa permitia o desenvolvimento de plantas com poucas folhas e ramos, com exceção das plantas de fáfia que estavam mais próximos à beira de mata e, portanto, sujeitas à maior intensidade luminosa durante o dia.

A penicilina é uma é uma planta herbácea de ocorrência em ecossistemas com fisionomias campestres (Luca et al., 2001). No entanto, no presente trabalho apresentou adaptação ao cultivo agroflorestal. Como as referências sobre os aspectos agronômicos dessa espécie, no Brasil, são muito escassas houve dificuldade de comparação dos resultados com outros trabalhos. Nas avaliações participativas realizadas com a comunidade, houve consenso de que o desenvolvimento dessa espécie estava de acordo com o conhecido popularmente.

A taxa de sobrevivência média observada na espinheira-santa, 16 meses pós-plantio foi de $70 \%$. Considerando-se apenas a área 1 esta taxa foi de $100 \%$, indicando alto nível de adaptação ao ambiente. Uma maior sobrevivência era esperada devido ao fato dessa espécie ser adaptada à condições de meia-sombra e ter sua ocorrência generalizada nos nas beiradas de matas de araucária, capões e em matas ciliares (Silva, 2003). Os valores obtidos para a altura média das plantas, aproximou-se dos valores médios obtidos por Rachwal et al. (1997) em que plantas de espinheirasanta com 2 anos e 1 mês de idade, atingiram valores de 37,95 e $13 \mathrm{~cm}$, para as alturas média, dominante e mínima, respectivamente. Benedetti et al. (2009) observaram alturas de 54,4 e 64,3 cm em plantas de espinheira santa aos 315 dias pós plantio sem e com adubação orgânica em casa de vegetação, o que indica que houve limitação ao crescimento devido à menor intensidade luminosa, uma vez que as plantas estavam adubadas.

A taxa de sobrevivência média observada para o patchuli e a altura das plantas de acordo com o citado por outros autores (Silva, 2003) 26 meses pós-plantio, faz dessa espécie a segunda mais adaptada às condições deste experimento (Tabela 4).

O guaco foi a espécie com a maior taxa média de sobrevivência ao final do experimento $(78 \%)$ e com comprimento médio dos ramos de $3,20 \mathrm{~m}$. Segundo Negrelle (1998) o guaco é uma das inúmeras plantas com potencial medicinal que está submetida à forte pressão extrativista. Sua adaptação ao cultivo em sistema agroflorestal é uma importante estratégia para reduzir o impacto engativo da exploração extrativista e permitir aos agricultores o fornecimento de um produto de forma sustentável.

A interação entre pesquisa, extensão, comunidade e outras instituições proporcionou maior segurança nos resultados, que foram discutidos e avaliados de forma conjunta em diversos momentos. $\mathrm{O}$ fato de o experimento ter sido desenvolvido dentro da comunidade fez com que houvesse interação com um grande número de famílias que o acompanharam através de dias de campo e outros eventos menores. Através do processo de pesquisa-participativa as famílias da comunidade puderam conhecer mais profundamente uma cadeia produtiva que eles supunham que podia ser uma boa alternativa de renda. Com a pesquisa, as famílias e os técnicos perceberam na prática, dificuldades como o acesso às áreas de plantio, as exigências do mercado, a necessidade de organização para atendê-las. Ou seja, além do conhecimento gerado ter sido apropriado em tempo real pelas pessoas

Rev. Bras. PI. Med., Campinas, v.15, n.4, p.774-779, 2013. 
envolvidas, aprofundou o nível de conhecimento da realidade da produção de plantas medicinais.

Apresentaram persistência ao cultivo em áreas sombreadas as espécies: Curcuma longa L.; Arctium lappa L.; Pfaffia glomerata (Spreng) Pedersen.; Mikania glomerata Sprengel.; Alternanthera sp.; Pogostemon cablin (Blanco) Benth. e Maytenus ilicifolia Mart. Ex Reissek indicando potencial para produção em sistemas agroflorestais.

\section{AGRADECIMENTOS}

Às famílias de Anair Cieslinski, Marta Marilda Cielusinski e Teodoro e Olinda Twardowski; à Associação da Comunidade de Rio Natal; ao STR de São Bento do Sul em nome de Gabriel Zigowski; ao ICMBio - Flona de Três Barras e ao Consórcio Quiriri (www.quiriri.com.br) de São Bento do Sul.

\section{REFERÊNCIA}

BENEDETTI, E.L. et al. Calagem e adubação no crescimento de espinheira-santa [Maytenus ilicifolia (Schrad.) Planch.] em casa de vegetação. Revista Brasileira de Plantas Medicinais, v.11, n.3, p.269276, 2009.

BRASIL. Lei n. 9.985, de 18 de julho de 2000. Regulamenta o art. 225, $\S 1^{\circ}$, inciso I, II, III e VII da Constituição Federal, institui o Sistema Nacional de Unidades de Conservação da Natureza e dá outras providências. Presidência da República, Casa Civil, Brasília, DF, 18 jul. 2000. Disponível em: <http://www. planalto.gov.br/ccivil_03/LEIS/L9985.htm>Acesso em: 05 ag. 2009.

CASTRO, E.M. et al. Adaptações anatômicas de folhas de Mikania glomerata Sprengel (Asteraceae), em três regiões distintas da planta, em diferentes níveis de sombreamento. Revista Brasileira de Plantas Medicinais, v.9, n.2, p.8-16, 2007.

GASSI, R.P. et al. Doses de fósforo e de cama-de-frango na produção de bardana. Ciência e Agrotecnologia. [online]. 2009, vol.33, n.3, pp. 692-697.

GUERREIRO, C.P.V. et al. Produção de $\beta$-ecdisona em
Pfaffia glomerata (Spreng.) Pedersen em função da adubação orgânica em 6 épocas de crescimento. Revista Brasileira de Plantas Medicinais, v.11, n.4, p.392-398, 2009.

HANISCH, A. L., MEISTER, L. A. Produção e qualidade da pastagem de Hemarthia altissima cv. Flórida em sistemas de produção de leite manejada com princípios agroecológicos. Revista Brasileira de Agroecologia, v.4, p.767 - 770, 2009.

LARCHER, W. Ecofisiologia Vegetal. São Carlos, Rima, 2000. 531p.

LORENZI, H.E.; MATOS, F.J.A. Plantas medicinais no Brasil: nativas e exóticas. Nova Odessa: Instituto Plantarum. 2002. 512 p.

LUCA, R.L. et al. Ação de diferentes faixas do espectro luminoso na otimização da produção de Alternanthera brasiliana L., uma planta medicinal. In: ENCUENTRO LATINOAMERICANO DE BIOTECNOLOGÍA VEGETAL, 4., 2001, Goiânia-GO. Anais... Goiânia: Redbio, 2001.6p

NEGRELLE, R.R.B. Exploração e comércio de produtos vegetais não madeiráveis: o caso das plantas medicinais. In: LIMA, R.X., NEGRELLE, R.R.B. Meio ambiente e desenvolvimento do litoral do Paraná: diagnóstico. Curitiba. Ed.UFPR, 1998. p. 65-92.

RACHWAL, M.F.G. CURCIO, G.R.; MEDRADO, M.J.S.. Desenvolvimento e produção de massa foliar de espinheira santa (Maytenus ilicifolia) a pleno sol , em cambissolo húmico no município de Colombo-PR. Boletim de Pesquisa Florestal, 35, p.91-93, 1997.

SCHÄFFER, W.B.; PROCHNOW, M. organiz. A Mata Atlântica e você: como preservar, recuperar e se beneficiar da mais ameaçada floresta brasileira. Brasília: Apremavi, 2002. 156p.: il.

SILVA JR, A.A. Essentia herba: Plantas Bioativas. Florianópolis:Epagri, 2003. 441p. v.1.

SILVA JR, A.A. Essentia herba: Plantas Bioativas. Florianópolis:Epagri, 2006. 633p. v.2.

STEENBOCK, W. Fundamentos para o manejo de populações naturais de espinheira-santa, Maytenus ilicifolia Mart. ex Reiss. (Celastraceae). 2003. 145f. Dissertação (Mestrado em Recursos Genéticos Vegetais) - Pós-graduação em Recursos Genéticos Vegetais, Universidade Federal de Santa Catarina

WHATLEY, F.H.; WHATLEY, F.R. A luz e a vida das plantas. São Paulo: EPU-EDUSP, 1982. 101 p. (Temas de Biologia, 30). 